\title{
Effect of Early Attachment on Psychological Development
}

\begin{abstract}
Huiqi Xu
Guangdong Guangya High School

Corresponding author.Email: huiqixu1205@outlook.com

ABSTRACT

The attachment theory was first proposed by British psychologist John Bowlby, outlining the emotional bonds between infancy and its caregivers and emphasizing the profound effect of this relationship on the future development of infancy. Further research has deepened this topic. To specifically understand the types of attachment and clarify the specific impact of early attachment on psychological development, this paper provides an overview of attachment theory and gives an analysis of the relationship. The result shows that attachment mainly can be divided into four types and early attachment has a deep influence on psychological development. Early attachment is mainly reflected in the baby's life experience. It includes positive and negative aspects. These effects are mainly reflected from biological, social and other aspects, and affect infants' psychological development by affecting their brain structure. Attachment is very important for children's early development. Through this study, society and family can correctly understand the specific impact of attachment on early childhood development, so as to pay more attention to the healthy development of early childhood.
\end{abstract}

Keywords: attachment, early experience, infancy, mental development, psychology.

\section{INTRODUCTION}

The caregiver-infancy relationship is seen to be the most important among all the relationships forming among life spans [1]. The attachment theory proposed by British psychologist John Bowlby laid the foundation on the research about the infant-caregiver relationship, which raised predictions and questions of attachment allowing further development [2]. Some studies also demonstrated the significance of early-stage connections, while others emphasize intrinsic factors such as temperament on the determinants of one's personality. Recent studies tend to consider both "nature" and "nurture" in researching human development by incorporating neurodevelopmental, psychosocial, and biological research [3]. Further studies have investigated the association between early quality and timing of emotional bonds with caregivers and the influence on psychological development. Some longitudinal studies also evaluate the underlying mechanism of the effect of different kinds of attachment, enabling researchers to develop the method of interventions of inappropriate infant-caregiver relationships.

This article synthesizes the existing study on attachment theories and the impact of different styles of attachment on mental development. The article discusses the development and refinement of attachment theories and their classification, different types of attachment and their impact on limbic, right brain and other neurological development. The social abilities and potential psychopathology influenced by certain attachment are also covered. The article arranges existing studies on this topic and points out the unsolved questions within this area of study including an organized guide of specific behaviors of certain attachment styles. This paper can lay a basic foundation for future research of the further and distinct classification among different attachments.

\section{ATTACHMENT THEORY}

\subsection{The Definition of Attachment}

The concept of "attachment" was first introduced by John Bowlby who was striving to understand the distress and anxiety of children separated from primary caregivers. Some earlier behaviorists suggested the attachment between children and caregivers was simply the result of feeding relationships [4]. According to Bowlby's observation, the anxiety in children didn't reduce even though they were fed. Then Bowlby found the attachment between children and caregivers had a 
clear motivation pattern as children sought proximity with their caregivers to receive comfort and care [4].

According to Bowlby, primary caregivers who can provide children with consistent responses allow children to develop a sense of security. When separated from the primary caregivers, children will feel distressed and anxious. Early bonds formed by children with their caregivers influence tremendously throughout life [5]. The attachment has adaptive value to ensure that children's psychological and physical needs can be met [6].

Bowlby also proposed the internal working model (IWMs), which refers to the quality of children's experience with their caregivers affecting children's cognitive-affective representations [7]. He argued that infants' and caregivers' interactions affect infants' expectations and ideas about themselves; infants learn to expect the appropriate responses from caregivers when needed after being responded consistently. While the proper response is not provided, the infant will distrust others and perceive themselves as not deserving of being treated well [7]. According to Bowlby, one's IWMs could be changed at a certain point. However, the transformations are limited by prior experiences with caregivers. The future development is dependent on the quality of the attachment with its caregiver [1].

Bowlby attributed the natural ties between children and caregivers to the evolutionary products, and the ability to connect with caregivers has been favored by natural selection [8]. As infants are vulnerable and helpless, they need to depend on a caregiver to enhance their chances to live and survive. In other words, when there is perceived danger or stress, for example, hunger, illness, pain, absence of parents, seeking physical proximity with caregivers can ensure the comfort and survival of the infant. Bowlby defined these behaviors of seeking closeness to caregivers driven by internal and environmental cues as the attachment system, comprising a series of attachment behavior such as reaching and following parents. The caregiving system is devised to respond to children's behaviors when parents detect the children's need to be cared for and protected [8].

\subsection{Classification of Attachment}

To examine the individual differences in attachment style, psychologist Mary Ainsworth designed the semistructured laboratory observations known as "Strange Situation Procedure" (SSP), which further developed attachment theory proposed by John Bowlby. The study observed the response of children aged 12 to 18 months when they were left alone at home and reunited with caregivers [4]. Based on the result of the study, Ainsworth synthesized attachment into three major types: secure attachment, ambivalent-insecure attachment, and avoidant-insecure attachment. Further studies done by Main and Simon (1986) demonstrated the fourth attachment named disorganized-insecure attachment.

\subsubsection{Secure Attachment}

According to Gearity (2005), a healthy infantcaregiver relationship must meet two basic needs: establishing basic trust of the world-consistent and timely responses to infant's need; allowance of emotional expressions along with underlying physiological patterning. The infant could form a satisfactory and secure relationship with the caregiver only if these two goals are achieved. Infants with these needs met are more likely to explore the surrounding environment with the guide of caregivers who give them care and protection. Consequently, children with these features can be more competent and independent in the future. According to Ainsworth et al., infants of secure attachment "show some protest" when being separated from primary caregivers or left alone with strangers in an unfamiliar environment "by the time of the first birthday". They will show obvious distress, disruption of normal behavior such as playing and exploring the surroundings, and rejecting the comfort of strangers [1].

\subsubsection{Ambivalent-Insecure Attachment}

Ambivalent-insecure attachment results from inconsistent and insufficient protection and care of caregivers. Lack of protection and care prevents infants from predicting if their expressions of anxiety and distress can be detected [1]. The infant is not confident about the availability of care and protection from caregivers, thus showing a low threshold for distress. When separated from caregivers, the ambivalentinsecure infant often cries even after the return of caregivers. Infants with this type of attachment are not easily comforted and don't show active reactions to settle from caregivers.

\subsubsection{Avoidant-Insecure Attachment}

Avoidant-insecure attachment developed when the caregiver rarely responds to the affection and distress of the infant and rarely shows sympathy and comfort. The avoidant-insecure infant barely cries during separation from the caregiver and rejects contact with his or her return [6]. Infants with this attachment do not show significant reactions, including pain or anxiety, to caregivers leaving unfamiliar environments. The infant even actively attempts to explore the surrounding after the caregiver's departure [1]. Moreover, the infant does not show particular affection to strangers or its caregiver and even avoids the caregiver's attention on the infant [1][7]. 


\subsubsection{Disorganized-Insecure Attachment}

In Main's Adult Attachment Interview (AAI), she found that parents of children with disorganized-insecure attachment always have unresolved emotional issues. While the child still attempts to seek proximity and safety from the caregiver, its parent leaves no space for giving adequate care and protection to the child (Firestone). Therefore, the infant loses the basic trust of interpersonal relations [1]. Disorganized-Insecure infants often show confusion and fear towards caregivers. They might actively react to the returns of caregivers but then ignore their existence, indicating the insecurity of the infants [6].

\section{RELATIONSHIP BETWEEN ATTACHMENT AND PSYCHOLOGICAL DEVELOPMENT}

\subsection{Early experience and brain development}

Many scientific studies proved that even though the genetic program initially generates abundant production of synaptic connections in the human brain, the environment selects the connections that are effectively necessitated by environmental information [9]. The infant's environmental experience either boosts or inhibits the structure and the functions of developing brains, which means that early interpersonal experience can influence the infant's "structural organization" of the brain and its developing adaptive functional abilities positively or negatively [9].

\subsubsection{Limbic System}

As the first two years of life is the critical period of development of the limbic system, early stress-regulating and emotion-charged experiences have a profound effect on later development [1][9]. A study done by Christina Moutsiana et al., investigated the effect of insecure attachment during infancy on amygdala volumes in early adulthood. They conducted a longitudinal study on a group of participants in which observed the attachment type of the infants at 18 months old then assessed them with MRI at 22 years. The result of the study shows that people with insecure attachment during infancy have greater amygdala volumes during early adulthood [10]. In addition, another research conducted by A RifkinGraboi (2015) further proved that insufficient maternal sensitivity is related to larger hippocampal volume during the infancy period [11]. Children with PTSD and maltreatment are also associated with increased volume of the hippocampus, which predisposes them to psychopathology [12].

Another remarkable translational study demonstrated that the absence of parental care was associated with the acceleration of maturation of fronto-amygdala connectivity. This was suggested to be mediated by cortisol levels, which was shown in institutionalized children, corresponding to the research finding on rodents [11][13]. The connectivity between hippocampus and brain regions important for memory is also studied. People who experienced higher maternal sensitivity have less connectivity between the hippocampus and regions involved in memory processing [11]. Higher maternal sensitivity means less connection between the left hippocampus and the left entorhinal cortex, and less connection between the left amygdala and the left entorhinal cortex, which is important for autobiography and affects the storage and extraction of memory as the interface between the hippocampus and the frontal cortex [11].

\subsubsection{The role of right brain}

The human's right brain is actively responsible for processing social-emotional information, promoting attachment functions, regulating bodily and affective states, and controlling functions sustaining survival and stress-coping mechanism [14][15][16]. A large body of evidence suggests that the first three years of human life is the crucial stage of maturation of the right brain [14]. Therefore, the early attachment experience is directly related to the development of the right brain.

Evidence has revealed that both the infant's and caregiver's right brains are involved in attachment relationships [14][15]. According to Perovich and Gewirtz, the learning process that mediates attachment is known as the synchrony between sequential infantmaternal stimuli and behavior. During this process, both the caregiver and the infant show sympathetic cardiac acceleration and then parasympathetic deceleration when responding to smiles from the other [17]. Both two brains cogenerating synchronized emotional communications with each other are involved in the dyadic process. Schore also suggested that when two right brain systems are mutually entrained in the synchrony, they can create a context of resonance that is essential in brain organization, central nervous system (CNS) regulatory process and the connectivity properties [18][19]. Trevarthen proposed that the resonance of the dyad facilitates the inter coordination of affective brain states. Contemporary neuroscientists refer resonance to as the capacity of neurons to respond selectively to inputs at certain frequencies, and "amplified resonance" serves as a basis for synchronizing patterns of network activity [19]. These principles can apply to interactions between the caregiver and the infant whose right hemisphere is involved in the analysis of facial, visual and auditory information of the caregiver [20]. Moreover, these "dyadically synchronized effectively charged transactions" stimulate a high level of metabolic energy for regulation of right-brain cortical and subcortical networks which process socioemotional information 
[14]. Simply speaking, interactions between caregivers and infants trigger stimulation in the right brain. The result of synchronized emotional transactions is that there is increased coherence in the infant's right brain as more energy flows between the higher right cortical and lower right subcortical components, which allow a higher level of complexity in the right brain [21].

\subsubsection{Other neurobiological evidence}

Investigations with PET brain scans on institutionalized Romanian children found comparably lower metabolism in the areas where are involved in stress regulation, including the orbitofrontal cortex [22]. There are also findings that suggest the hypoactivation of right-hemisphere frontal, medial temporal and limbic structures participated in emotion regulations leads to integration and connectivity between these regions in children who have experienced attachment-related trauma [23].

A 9-year longitudinal study finds that better quality mother-child relationships in infancy predict larger GM volume in the superior and middle temporal gyri, superior temporal sulci, temporoparietal junction and precentral gyri in late childhood [24], which corresponded to the studies finding smaller GM volume or surface in the middle and superior temporal gyri of children who have experienced maltreatment [25][26][27][28]. These areas are essential in processing emotional stimuli [29]. A reduction of corpus callosum volumes in people with experience of abuse or neglect is discovered, specifically in the middle and posterior regions [30][31]. The abnormal interhemispheric connections are also related to emotional recognition, expression, as well as arousal impairments [32]. Furthermore, the HPA axis in children who have experienced inappropriate attachment is found to be dysregulated, where feedback loops that regulate glucocorticoid hormones are disrupted by constantly unpredictable or adverse attachment experiences [33], resulting in high sensitivity to stress [34].

\subsection{Potential Psychopathology and Social Functions}

Several studies have all pointed that insecure attachment at an early age can result in retarded social, neural and mental development, and a series of potential mental disorders. Bowlby suggests that maternal deprivation can result in a series of negative consequences including retarded growth, aggressiveness, dependency anxiety, intellectual retardation, social maladjustment, no feelings for other, depression and delinquency [1]. Societal interactions nourish infants' neural networks in their brains. Insufficiency of reliable interactions between infants and caregivers prevents growth of neural connections in infants' brains, and thus results in retarded, deficient brain and social development. Attachment children are more vulnerable to problems related to peer pressure or setback in daily life [35], which lead to serious self-doubt, and a pathological state of anxiety [36]. Research has shown that the infant who grows up in an unresponsive, insecure environment will tend to have constant feelings of vulnerability, and anxiety, increasing the risk of having anxiety disorder [37][1]. People who failed to be nourished by secure attachment will have difficulties in relations throughout life. To be more specific, an inadequate basic sense of trust may inhibit a child from leaving the caregiver to explore the surroundings, which decreases opportunities for him or her to become competent and learn about the world. On the contrary, as secure-attachment people form the basic trust of the world, they are more likely to explore their surroundings and learn about the world.

Although histories of secure attachment cannot ensure healthy functioning in the future, the number of those who experienced secure attachment had problems at any given age was significantly fewer than those with histories of resistant or avoidant histories particularly those with disorganized-attachment experience [38]. Moreover, when children with troubling behavior, either in the preschool years or middle childhood, followed into the period of recovery, the extent of their recoveries was predicted by the history of secure attachment and nourishment. Disorganized attachment during infancy strongly predicted later disturbance. According to Scroufe, self-injurious behavior (SIB) is a likely precursor of personality disorders despite lacking data fully proving such a link [38]. The data has shown that SIB (e.g, cutting, burning) in early adulthood is tightly connected with a history of disorganized attachment or maltreatment especially sexual abuse [38].

\section{DISCUSSION}

Despite existing studies have delineated the relations between attachment and its consequences, the factors contributing to different styles of attachment and the mechanism aiming to intervene in improper attachment relationships haven't been clearly investigated. Future research will focus on this direction. Although attachment theory provides the basic framework of attachment styles, more detailed actions and reactions between the infant and the caregiver haven't been studied, hindering the development of early interventions in improper attachment relationships. Future studies related to this topic can concentrate on uncovering the factors leading to different types of attachment and establishing organized guidance to intervene in improper attachment relationships. 


\section{CONCLUSION}

The result shows that the life experience in the first three years is essential for future development including neurobiological, social and emotional development. Both positive and negative experiences influence the infant's brain structure and thus psychological development in the future. Children growing in an enriched environment with the consistent and sensitive responses provided are likely to become competent and confident in the future. Conversely, children with poor nourishment and inconsistent and insensitive response from caregivers will predispose them to behavioral and psychopathological problems. This paper can provide a theoretical basis for a better understanding of the influencing factors of children's early healthy development. The limitation of this paper is that the number of literature based on research and analysis is relatively small. Future research should expand the scope of literature selection and make a more comprehensive summary and analysis from more angles and more entry points.

\section{REFERENCES}

[1] Mokhtar Malekpour. Effects of Attachment on Early and Later Development, The British Journal of Development Disabilities, 53:105, 2007, pp.81-95, DOI: $10.1179 / 096979507799103360$

[2] Ainsworth, M. D. S. Infancy in Uganda: Infant Care and the Growth of Attachment. Baltimore, MD: Johns Hopkins University Press, 1967, 1.

[3] Louise Newman, Carmel Sivaratnam \& Angela Komiti. Attachment and early brain development neuroprotective interventions in infant-caregiver therapy, Translational Developmental Psychiatry, 3:1, 2015, 28647, DOI: 10.3402/tdp.v3.28647

[4] Cherry, K. (2019). The Story of Bowlby, Ainsworth, and Attachment Theory. [online] Verywell Mind. Available at: https://www.verywellmind.com/whatis-attachment-theory-2795337.

[5] Bowlby, J. (1973). Attachment and loss. Vol.2: Separation: Anxiety and anger. New York: Basic Books.

[6] Papalia, D.E., Olds, S.W. and Feldman, R.D. A child's world: Infancy through adolescence. 1999, pp.245-252. The McGrow Hill Companies, Inc. New York, USA.

[7] Fransson, M. 2014. Attachment and the Development of Personality and Social Functioning. Digital Comprehensive Summaries of Uppsala Dissertations from the Faculty of Social Sciences 79 pp. Uppsala: Acta Universitatis Upsaliensis.
[8] Bowlby, J. (1969). Attachment and loss. Vol.1: Attachment (P.214). New York: Basic Books.

[9] Schore, A. N. Effects of a secure attachment relationship on right brain development, affect regulation, and infant mental health. Infant Mental Health Journal, 22(1-2), 2001, pp.7-66. doi:10.1002/1097-0355(200101/04)22:1<7::aidimhj2>3.0.co;2-n

[10] Moutsiana, Christina; Johnstone, Tom; Murray, Lynne; Fearon, Pasco; Cooper, Peter J.; Pliatsikas, Christos; Goodyer, Ian; Halligan, Sarah L. Insecure attachment during infancy predicts greater amygdala volumes in early adulthood. Journal of Child Psychology and Psychiatry, 56(5), 2015, pp. 540-548. doi:10.1111/jcpp.12317

[11] Rifkin-Graboi, A., Kong, L., Sim, L. W., Sanmugam, S., Broekman, B. F., Chen, H., et al. (2015). Maternal sensitivity, infant limbic structure volume and functional connectivity: a preliminary study. Transl. Psychiatry 5:e668 doi: 10.1126/science. 1252723

[12] Qiu A, Rifkin-Graboi A, Chen H, Chong YS, Kwek $\mathrm{K}$, Gluckman PD et al. Maternal anxiety and infants hippocampal development: timing matters. Nat Transl Psychiatry 2013; 3: e306.

[13] Gunnar MR, Morison SJ, Chisholm K, Schuder M . Salivary cortisol levels in children adopted from romanian orphanages. Dev Psychopathol 2001; 13: pp. 611-628.

[14] Schore, A.N. (1994). Affect regulation and the origin of the self: The neurobiology of emoti- onal development. Mahwah, New Jersey. Erlbaum.

[15] Schore, A.N. (1998). The experience-dependent maturation of an evaluative system in the cortex. In: K.

[16] Wittling, W., \& Schweiger, E. Neuroendocrine brain asymmetry and physical complaints. Neuropsychologia, 31, 1993, pp.591-608.

[17] Donovan, W.L., Leavitt, L.A., \& Balling, J.D. Maternal physiological response to infant signals. Psychophysiology, 15, 1978, pp. 68-74.

[18] Salansky, N., Fedotchev, A., \& Bondar, A. Responses of the nervous system to low frequency stimulation and EEG rhythms: Clinical implications. Neuroscience and Biobehavioral Reviews, 22, 1998, pp.395 - 409.

[19] Schore, A. N. Effects of a secure attachment relationship on right brain development, affect regulation, and infant mental health. Infant Mental Health Journal, 22(1-2), 2001, pp.7-66. 
doi:10.1002/1097-0355(200101/04)22:1<7::aid-

imhj2>3.0.co;2-n

[20] Ornstein, R. (1997). The right mind: Making sense of the hemispheres. New York: Harcourt Brace

[21] van Pelt, J., Corner, M.A., Uylings, H.B.M., Lopes da Silva, F.H. (Eds.). (1994). Progress in brain research, the self-organizing brain: From growth cones to functional networks, vol. 102. Amsterdam: Elsevier.

[22] Chugani HT, Behen ME, Muzik O, Juhasz C, Nagy F, Chugani DC. Local brain functional activity following early deprivation: a study of postinstitutionalized Romanian orphans. NeuroImage 2001; 14: 1290 $\square 301$.

[23] Schore, A. N. (2009). Right-brain affect regulation: An essential mechanism of development, trauma, dissociation, and psychotherapy. In D. Fosha, D. J. Siegel, \& M. F. Solomon (Eds.), The healing power of emotion: Affective neuroscience, development \& clinical practice (pp. 112-144). W. W. Norton \& Company.

[24] Leblanc É, Dégeilh F, Daneault V, Beauchamp MH and Bernier A (2017) Attachment Security in Infancy: A Preliminary Study of Prospective Links to Brain Morphometry in Late Childhood. Front. Psychol. 8:2141. doi: 10.3389/fpsyg.2017.02141

[25] De Brito, S. A., Viding, E., Sebastian, C. L., Kelly, P. A., Mechelli, A., Maris, H., et al. Reduced orbitofrontal and temporal grey matter in a community sample of maltreated children. J. Child Psychol. Psychiatry 54, 2013, pp. 105-112. doi: 10.1111/j.1469-7610.2012.02597.x

[26] Kelly, P. A., Viding, E., Wallace, G. L., Schaer, M., De Brito, S. A., Robustelli, B., et al. Cortical thickness, surface area, and gyrification abnormalities in children exposed to maltreatment: neural markers of vulnerability? Biol. Psychiatry 74, 2013, pp.845-852. doi: 10.1016/j.biopsych.2013.06.020

[27] Kelly, P. A., Viding, E., Puetz, V. B., Palmer, A. L., Mechelli, A., Pingault, J. B., et al. Sex differences in socioemotional functioning, attentional bias, and gray matter volume in maltreated children: a multilevel investigation. Dev. Psychopathol. 27(4 Pt 2), 2015, pp.1591-1609. doi: $10.1017 /$ S0954579415000966

[28] Lim, L., Radua, J., and Rubia, K. Gray matter abnormalities in childhood maltreatment: a voxelwise meta-analysis. Am. J. Psychiatry 171, 2014, pp. 854-863. doi: 10.1176/appi.ajp.2014.13101427
[29] Allison, T., Puce, A., and McCarthy, G. Social perception from visual cues: role of the STS region. Trends Cogn. Sci. 4, 2000, pp.267-278. doi: 10.1016/S1364- 6613(00)01501- 1

[30] Kitayama N, Brummer M, Hertz L, Quinn S, Kim Y, Bremner JD. Morphologic alterations in the corpus callosum in abuse- related posttraumatic stress disorder: a preliminary study. J Nerv Ment Dis 2007; 195: $1027 \square 9$.

[31] Jackowski AP, Douglas-Palumberi H, Jackowski M, Win L, Schultz RT, Staib LW, et al. Corpus callosum in maltreated children with posttraumatic stress disorder: a diffusion tensorimaging study. Psychiatry Res 2008; 162: 256 $\square 61$

[32] Hooker C, Park S. Emotion processing and its relationship to social functioning in schizophrenia patients. Psychiatry Res 2002; 112: $41 \square 50$.

[33] Burke HM, Davis MC, Otte C, Mohr DC. Depression and cortisol responses to psychological stress: a meta-analysis. Psychoneuroendocrinology 2005; 30: $846 \square 56$.

[34] Kaufman J, Charney D. Effects of early stress on brain structure and function: implications for understanding the relationship between child maltreatment and depression. Dev Psychopathol 2001; 13: 451 $\square 71$.

[35] Barnett, D. and Butler, C.M. Atypical patterns of early attachment: Discussion and future directions. Monographs of the Society of Research in Child Development, 64, 1999, pp.172-191.

[36] Thompson, R.A. New directions for child development in the twenty-first century: The legacy of early attachments. Child Development, 71, 2000, pp. 145-152.

[37] Warren, S.L., Huston, L., Egeland, B. and Sroufe, L.A. Child and adolescent anxiety disorders and early attachment. Journal of the American Academy of Child and Adolescent Psychiatry, 36, 1997, pp. 637-644.

[38] Sroufe, L. Alan. Attachment and development: A prospective, longitudinal study from birth to adulthood. Attachment \& Human Development, 7(4), 2005, pp.349-367. doi:10.1080/14616730500365928 